\title{
Upregulation of OLR1 and IL17A genes and their association with blood glucose and lipid levels in femoropopliteal artery disease
}

\author{
CANER ARSLAN ${ }^{1 *}$, BURCU BAYOGLU $^{2 *}$, CIGDEM TEL $^{1}$, \\ MUJGAN CENGIZ ${ }^{2}$, AHMET DIRICAN ${ }^{3}$ and KAZIM BESIRLI ${ }^{1}$
}

\begin{abstract}
Departments of ${ }^{1}$ Cardiovascular Surgery and ${ }^{2}$ Medical Biology, Cerrahpasa Medical Faculty, Istanbul University, Istanbul $34098 ;{ }^{3}$ Department of Biostatistics and Medical Informatics, Istanbul Medical Faculty, Istanbul University, Istanbul 34093, Turkey
\end{abstract}

Received April 4, 2016; Accepted October 28, 2016

DOI: 10.3892/etm.2017.4081

\begin{abstract}
Oxidized low-density lipoprotein receptor 1 (OLR1) and interleukin 17A (IL17A) have pro-inflammatory roles in the development of cardiovascular disorders. The present study evaluated the association of OLR1 and IL17A and their polymorphisms with the development of femoropopliteal (FP) artery disease. The mRNA expression of OLR1 and IL17A in peripheral blood mononuclear cells as well as the frequency of OLR1 rs11053646 and IL17A rs8193037 and rs3819025 polymorphisms were assessed by polymerase chain reaction in 70 patients with FP artery disease and 80 age-matched disease-free controls. Furthermore, the levels of plasma cytokines were assessed by multiplex immunoassay. OLR1 and IL17A mRNA expression was significantly higher in patients with FP artery disease compared with that in controls $(\mathrm{P}<0.001)$. No significant difference was observed in the genotypic frequencies of OLR1 rs11053646 $(\mathrm{P}=0.87)$ or in IL17A rs8193037 and rs3819025 $(\mathrm{P}=0.80$ and 0.92 , respectively) polymorphisms between patients with FP artery disease and controls. Plasma IL4, -6, -10, -22, -31 and -33 as well as soluble cluster of differentiation 40 ligand and tumor necrosis factor- $\alpha$ levels were significantly increased among FP artery disease patients compared with controls $(\mathrm{P}<0.05)$. Furthermore, OLR1 expression was positively correlated with triglyceride $(\mathrm{r}=0.463, \mathrm{P}<0.001)$, low-density lipoprotein cholesterol $(\mathrm{r}=0.507, \mathrm{P}<0.001)$ and total cholesterol levels $(r=0.357, P=0.006)$ in patients with FP artery disease. To the best of our knowledge, the present study was the first to
\end{abstract}

Correspondence to: Dr Burcu Bayoglu, Department of Medical Biology, Cerrahpasa Medical Faculty, Istanbul University, 53 Kocamustafapasa Road, Istanbul 34098, Turkey

E-mail: burcu.bayoglu@istanbul.edu.tr

*Contributed equally

Key words: peripheral artery disease, femoropopliteal artery occlusion, oxidized low-density lipoprotein receptor 1, interleukin 17A, plasma cytokine levels identify an association between OLR1 and IL17A genes and FP artery disease. OLR1 and IL17A mRNA transcripts may be associated with blood lipid parameters and with the development of FP artery disease.

\section{Introduction}

Peripheral artery disease (PAD) is an occlusive disease of the peripheral circulation system. PAD is one of the major syndromes of atherothrombosis and is found in $15-20 \%$ of individuals aged $>70$ years (1). Early diagnosis is important to improve patient quality of life and prevent secondary vascular events, such as stroke or acute myocardial infarction (AMI) (2). The presence of the occlusion is effectively measured by the ankle-brachial index (ABI), a non-invasive technique (2). Intermittent claudication in the lower limbs is a common clinical symptom of PAD and at present, the most effective treatment for occluded arteries is conventional surgery (2). There are a number of common risk factors for PAD, including age, diabetes mellitus, hypertension, smoking and hyperlipidemia (3). However, the development of PAD may also be independently influenced by genetic factors (4). Atherosclerosis accounts for $>90 \%$ of PAD cases in the United States (3).

Central lipid core, connective tissue, inflammatory cells and smooth muscle cells (SMCs) form plaques covered by a fibrous cap, which are characteristic of atherosclerotic lesions. Atherosclerotic plaques are typically located at the bifurcations or proximal segments of large- and medium-sized arteries (3). The popliteal and femoral arteries are affected in $80-90 \%$ of symptomatic PAD patients, the tibial and peroneal arteries are affected in $40-50 \%$ of patients and the aorto-iliac arteries are affected in $30 \%$ of patients (3).

Oxidized low-density lipoprotein receptor 1 (OLR1), also known as LOX1, has a pro-inflammatory role in atherogenesis. Lipoprotein modification in the arteries causes the peroxidation of lipids and generates aldehyde products, such as malondialdehyde. Lipid peroxidation induces inflammatory processes in vascular cells and inflammatory mediators accelerate the uptake of lipoprotein-derived lipids. Modified lipoproteins, including acetylated low-density lipoprotein (LDL) and oxidized LDL (oxLDL), cannot be detected by 
native LDL receptors. Macrophage receptor families recognize these modified lipoproteins and facilitate the formation of lipid-filled macrophages. oxLDL is self-promoting and pro-inflammatory cytokines are produced alongside the formation of more oxLDL through an OLR1-mediated cycle. The binding of oxLDL to OLR1 leads to an increase in intracellular reactive oxygen species (ROS). The elevated ROS level may cause superoxide anions to react with intracellular nitric oxide, resulting in endothelial dysfunction $(5,6)$.

Interleukin 17 (IL17) is a unique cytokine that has six isoforms (A-F) produced by a novel T helper (Th) subset, Th17 cells, and other cells in the immune system. IL17A has been studied as part of numerous inflammatory diseases $(7,8)$ and has been demonstrated to serve an essential role in the maintenance of angiotensin II-induced hypertension and vascular dysfunction (9). Another study has indicated that IL17A gene variants are significantly associated with an increased risk of developing coronary artery disease (CAD) and that IL17A is overexpressed in patients with AMI (10). In a recent study, oxLDL, a hyperlipidemia stimulus, was found to upregulate IL17 receptors in human primary aortic cells (11).

The OLR1 gene, located on chromosome 12p13.1-p12.3, spans over $7 \mathrm{~kb}$ and consists of six exons. A single nucleotide polymorphism (SNP) at rs11053646 (c.501 G>C) on exon 4 resulting in a lysine $(\mathrm{K})$ to asparagine $(\mathrm{N})$ amino acid substitution at position 167 (p.K167N) has been identified to be associated with the decreased binding and internalization of oxLDL (12). Another SNP at rs11053646 was found to be associated with hypertension, MI and carotid atherosclerosis $(13,14)$ as well as ischemic stroke $(15-17)$.

The IL17A SNP rs8193037 (-121 G>A) and IL17A SNP rs3819025 (+45 G>A) are located in the 5' region of intron 1 of the IL17A gene. It has been demonstrated that IL17A gene variants are associated with CAD (10).

The OLR1 and IL17A genes may jointly impact the phenotype during the development of PAD. Investigating the possible association between OLR1 and IL17A gene expression and OLR1 rs11053646 and IL17A rs8193037 and rs3819025 variants may therefore help to determine the pathogenesis of PAD. To the best of our knowledge, no studies have yet examined the association of OLR1 and IL17A with femoropopliteal (FP) artery disease, a sub-type of PAD. Due to OLR1 and IL17A exhibiting a functional significance in atherogenesis, the present study assessed the mRNA expression and frequency of OLR1 rs11053646 and IL17A rs8193037 and rs3819025 polymorphisms, as well as the levels of Th17-associated cytokines in a sample of Turkish patients with FP artery disease. It was then evaluated whether mRNA expression of OLR1 and IL17A was associated with peripheral circulation and its pathology.

\section{Materials and methods}

Patients. OLR1 and IL17A mRNA levels, OLR1 rs11053646 and IL17A rs8193037 and rs3819025 genotypes as well as plasma cytokine levels were compared between patients diagnosed with FP artery disease and healthy controls. The present study included 150 Turkish patients, consisting of 70 patients with FP artery disease (50 male, 20 female; mean age, $61.76 \pm 10.95$ years; range, $42-82$ years) and 80 healthy controls (56 male, 24 female; mean age, 60.61 \pm 6.34 years; range, 50-78 years) recruited at the Cardiovascular Surgery Department at the Istanbul University Cerrahpasa Medical Faculty between January 2015 and January 2016. The present study was conducted according to the principles of the Declaration of Helsinki and was approved by the Local Ethics Committee of the Cerrahpasa Medical Faculty, Istanbul University (Istanbul, Turkey). Prior to participation, all participants provided written informed consent.

Eligible patients were recruited on the basis of atherosclerotic occlusions of the FP peripheral arteries $(\geq 50 \%)$ detected by physical examination, duplex Doppler ultrasound, ankle brachial index (ABI), magnetic resonance angiography, computed tomography-angiography and digital subtraction angiography. Exclusion criteria for the patient group were presence of acute or chronic inflammatory disease, immunological disease, cancer and pregnancy. All subjects with FP artery disease underwent surgery at the Cardiovascular Surgery department of the Cerrahpasa Medical Faculty, Istanbul University, which was scheduled following blood tests; all of the patients received statin treatment.

The control group consisted of 80 healthy individuals who visited the Cerrahpasa Medical Faculty Hospital (Istanbul, Turkey) for regular health screening without any clinical findings of PAD and were randomly selected. Inclusion criteria were no use of statins and a normal lipid profile. The exclusion criteria included the presence of cardiovascular disease, severe kidney and hepatic diseases, diabetes, cancer, hypertension, autoimmune diseases, pregnancy and any atherosclerosis risk factor such as obesity, smoking or a family history of cardiovascular disease. Furthermore, healthy controls were excluded from the study if intermittent claudication with palpable pulses on their lower extremity arteries was identified upon examination.

Blood samples and DNA extraction. In order to isolate DNA, venous blood samples from all participants were collected into EDTA tubes and stored at $-20^{\circ} \mathrm{C}$ in aliquots until use. Genomic DNA was extracted from whole blood using a high pure PCR template preparation kit (Roche Diagnostics $\mathrm{GmbH}$, Mannheim, Germany) according to the manufacturer's protocol.

Blood samples and isolation of peripheral blood mononuclear cells (PBMCs) and RNA. Venous blood samples obtained from all participants were collected into heparin tubes and immediately underwent RNA extraction and lymphocyte separation. Using the PureLink ${ }^{\circledR}$ RNA Mini kit (Thermo Fisher Scientific, Inc., Waltham, MA, USA) total RNA was extracted from freshly isolated PBMCs according to the manufacturer's protocol.

Reverse transcription-quantitative polymerase chain reaction $(R T-q P C R)$. Following RNA extraction, $400 \mathrm{ng}$ of total RNA was reverse-transcribed into complementary (c)DNA with random hexamers as primers using the Transcriptor High Fidelity cDNA Synthesis kit (Roche Diagnostics), according to the manufacturer's protocol. OLR1 and IL17A expression in PBMCs was determined by qPCR using a LightCycler ${ }^{\circledR} 1.5$ detection system(Roche Applied Science,Pleasanton,CA,USA) with TaqMan probe technology (TIB Molbiol GmbH, Berlin, 
Germany). cDNA samples were amplified with hydrolysis probes in qPCR reactions for pre-incubation at $95^{\circ} \mathrm{C}$ for $10 \mathrm{~min}$, 45 cycles of denaturation at $95^{\circ} \mathrm{C}$ for $10 \mathrm{sec}$, annealing at $60^{\circ} \mathrm{C}$ for $30 \mathrm{sec}$ and extension at $72^{\circ} \mathrm{C}$ for $1 \mathrm{sec}$, followed by cooling at $40^{\circ} \mathrm{C}$ for $30 \mathrm{sec}$. OLR1 (catalogue no. 05532957001-90015528) and IL17A (catalogue no. 05532957001-90015530) mRNA levels were normalized to endogenous reference genes: ACTB (catalogue no. 05532957001-90018066), B2M (catalogue no. 05532957001-90010199) and GAPDH (catalogue no. 05532957001-90015529) using the $2^{-\Delta \Delta \mathrm{Cq}}$ method (18). The primers and probes were chosen from Roche UPL system (Roche Diagnostics GmbH, Mannheim, Germany). Three endogenous stably expressed reference genes were used to prevent erroneous normalization. Primers were designed as follows: $A C T B$, forward 5'-AGAGCTACGAGCTGCCTG AC-3' and reverse 5'-CGTGGATGCCACAGGACT- 3'; and $B 2 M$, forward 5'-ATCTGAGCAGGTTGCTCCAC-3' and reverse 5'-GACCAAGATGTTGATGTTGGATAA-3'. The amplicon lengths of OLR1, IL17A, ACTB, B2M and GAPDH were $62,69,114,95$, and $66 \mathrm{nt}$, respectively. $\mathrm{Cq}$ values of 40 were excluded from the study. The experiments were performed twice.

Genotyping of OLR1 rs11053646 and IL17A rs8193037 and rs3819025 SNPs. OLR1 rs11053646 (catalogue no. 29931401) and IL17A rs8193037 (catalogue no. 31931501) and rs3819025 (catalogue no. 32321401) SNPs (TIB Molbiol GmbH) were determined using the LightCycler ${ }^{\circledR} 1.5$ detection system with hybridization probes consisting of 3'-fluorescein and a 5'-LightCycler ${ }^{\circledR}$ Red-labeled pair of oligonucleotide probes (TIB Molbiol GmbH). Genotyping was performed using a total reaction volume of $20 \mu \mathrm{l}$ containing $1.0 \mu \mathrm{l}$ primer-probe mix $2.0 \mu \mathrm{l} \mathrm{LightCycler}{ }^{\circledR}$ FastStart DNA Master HybProbe (Roche Diagnostics $\mathrm{GmbH}$ ), $3.0 \mathrm{mM}$ magnesium chloride and $50 \mathrm{ng}$ genomic DNA. The melting temperature profiles and the results of melting curve analysis were used to identify the genotype of PCR products. The quality of SNP genotyping was verified by independent replications of the genotyping, using randomly selected samples; quality control results agreed with the initial genotyping results.

Multiplex immunoassay. The levels of the plasma cytokines IL1B, -4, -6, -10, -17A, -17F, -21, -22, -25, -31 and -33 as well as interferon- $\gamma$ (IFNG), soluble cluster of differentiation 40 ligand (SCD40L) and tumor necrosis factor- $\alpha$ (TNF- $\alpha$ ), were measured using the Bio-Plex ${ }^{\circledR}$ system (catalogue no. 171-AA001M; Bio-Rad Laboratories, Inc., Hercules, CA, USA) according to the manufacturer's protocol. The 96-well plates were prepared with assay buffer (Bio-Rad Laboratories, Inc.). Controls, standards and samples to be analysed were added (total volume, $50 \mu \mathrm{l}$ ) to the wells and incubated with antibody-immobilized microbeads (capture antibody; 1:20) for $1 \mathrm{~h}$ at room temperature (catalogue no. 171-AA001M, Bio-Rad Laboratories, Inc.). Following washing, biotinylated detection antibodies at a dilution of 1:20 (anti-human IL1B, -4, -6, -10, $-17 \mathrm{~A},-17 \mathrm{~F},-21,-22,-25,-31,-33$, IFNG, SCD40 L, and TNF- $\alpha$ antibodies; catalogue no. 171-AA001 M, Bio-Rad Laboratories, Inc.) were incubated in the dark for $30 \mathrm{~min}$ at $\mathrm{RT}$ with the bound cytokines. Fluorescent (phycoerythrin-labeled) streptavidin (1:100; Bio-Rad Laboratories, Inc.) was added.
A final wash was completed prior to resuspension in sheath fluid for analysis in the Bio-Plex ${ }^{\circledR}$ array reader using the Bio-Plex Manager 4.1 software (Bio-Rad Laboratories, Inc.). The concentration of each cytokine $(\mathrm{pg} / \mathrm{ml})$ was calculated against a standard curve plotted using a five-parameter logistic regression.

Statistical analysis. Continuous variables were compared between healthy controls and patients with FP artery disease using the Student's t-test or Mann-Whitney U Test; data were expressed as the mean \pm standard deviation. For categorical variables, the $\chi^{2}$ test or two-sided Fisher's exact test were used. These included genotype and allele frequencies to compare the association between genotypes and alleles among cases and controls and to test the deviation of genotype distribution from the Hardy-Weinberg equilibrium (HWE). P $\leq 0.05$ was considered to indicate a statistically significant difference. To determine the strength of the association between genotypes, alleles and case/control status, odds ratios (OR) and their 95\% confidence intervals (CIs) were calculated. Furthermore, ORs and 95\% CI of SNP and gene expression levels were estimated by multiple logistic regression analysis with adjustments for age, gender, serum CRP levels, LDL cholesterol and hypertension status. Relative gene expression levels and biochemical parameters were compared using the Spearman's non-parametric correlation test. All statistical analyses were performed using SPSS software for Windows, version 21.0 (IBM SPSS Inc., Armonk, NY, USA).

\section{Results}

Demographic data. The demographic and clinical characteristics of healthy controls and patients with FP artery disease are presented in Table I. There were no statistically significant differences observed between the groups with regard to gender $(\mathrm{P}=0.86)$ or age $(\mathrm{P}=0.44)$. Patients with FP artery disease and controls exhibited significant differences in hematocrit $(\mathrm{Z}=-4.508, \mathrm{P}<0.001)$, fasting glucose $(\mathrm{Z}=-5.563, \mathrm{P}<0.001)$, urea $(Z=-2.934, P=0.003)$, creatinine $(Z=-3.477, P=0.001)$ and serum $C$-reactive protein $(\mathrm{CRP} ; \mathrm{Z}=-6.133, \mathrm{P}<0.001)$ levels but not in aspartate transaminase, alanine transaminase, total high-density lipoprotein (HDL) and LDL cholesterol or triglyceride levels $(\mathrm{P}>0.05$; Table I).

OLR1 and IL17A mRNA levels. OLR1 and IL17A mRNA levels were significantly higher in patients with FP artery disease $(Z=-4.114)$ compared with those in the healthy controls $(\mathrm{Z}=-5.679, \mathrm{P}<0.001$; Fig. 1).

OLR1 and IL17A mRNA levels were compared in male and female patients. Male patients exhibited higher IL17A mRNA levels compared with those in female subjects; however, this difference was not statistically significant $(\mathrm{P}>0.05$; results not shown).

Plasma cytokine levels. Plasma levels of IL4, -6, -10, 17A, -22, $-31,-33$ as well as SCD40 L and TNF- $\alpha$ were significantly higher in patients with FP artery disease, compared with the controls $(\mathrm{P}<0.05$; Fig. 2). In addition, plasma IL1B and IFNG levels were higher in patients with FP artery disease, but the difference was not significant ( $P>0.05$; Fig. 2). 
Table I. Demographic and clinical characteristics of patients with FP artery disease and controls.

\begin{tabular}{lccc}
\hline Characteristic & FP artery disease $(\mathrm{n}=70)$ & Control $(\mathrm{n}=80)$ & P-value \\
\hline Age (years) & $61.76 \pm 10.95$ & $60.61 \pm 6.34$ & 0.44 \\
Gender, M/F (\%) & $50 / 20(71.4 / 28.6)$ & $56 / 24(70.0 / 30.0)$ & 0.86 \\
Hematocrit, \% & $35.93 \pm 6.37$ & $40.73 \pm 4.37$ & $<0.001^{\mathrm{a}}$ \\
Fasting glucose, mg/dl & $141.25 \pm 67.20$ & $94.77 \pm 31.40$ & $<0.001^{\mathrm{a}}$ \\
AST, U/L & $22.59 \pm 14.83$ & $19.55 \pm 5.96$ & 0.123 \\
ALT, U/L & $20.82 \pm 15.35$ & $21.75 \pm 8.82$ & 0.669 \\
T cholesterol, mg/dl & $186.47 \pm 39.27$ & $192.37 \pm 35.25$ & 0.430 \\
HDL cholesterol, mg/dl & $41.64 \pm 16.48$ & $46.60 \pm 17.65$ & 0.141 \\
LDL cholesterol, mg/dl & $131.59 \pm 95.96$ & $132.54 \pm 32.75$ & 0.949 \\
Triglycerides, mg/dl & $180.21 \pm 219.02$ & $171.85 \pm 68.07$ & 0.81 \\
Urea, mg/dl & $45.34 \pm 27.57$ & $32.89 \pm 10.28$ & $0.003^{\mathrm{a}}$ \\
Creatinine, mg/dl & $1.19 \pm 1.17$ & $0.82 \pm 0.23$ & $0.001^{\mathrm{a}}$ \\
C-Reactive protein, $\mathrm{nmol} / \mathrm{l}$ & $46.92 \pm 70.21$ & $3.99 \pm 4.28$ & $<0.001^{\mathrm{a}}$ \\
\hline
\end{tabular}

Values are expressed as the mean \pm standard deviation unless otherwise indicated. ${ }^{a} \mathrm{P} \leq 0.05$; Student's t-test, Mann-Whitney U test, and $\chi^{2}$ test were performed. M/F, male/female; FP, femoropopliteal; AST, aspartate transaminase; ALT, alanine transaminase; T cholesterol, total cholesterol; HDL, high-density lipoprotein; LDL, low-density lipoprotein.
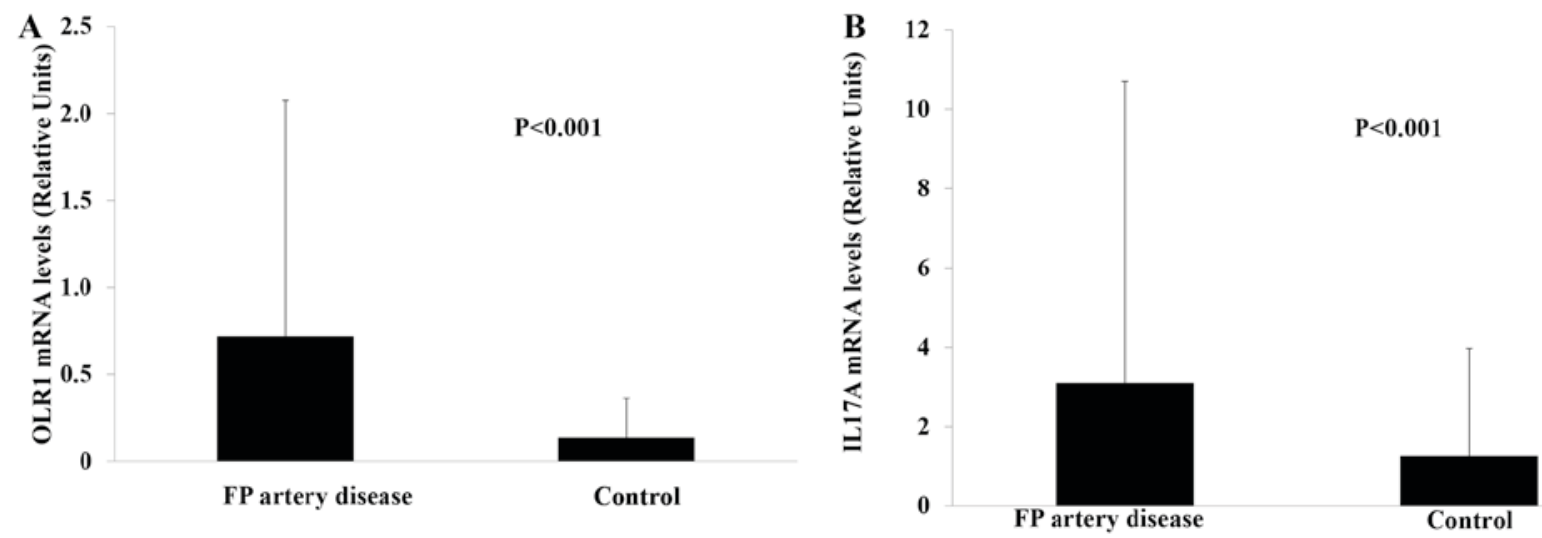

Figure 1. Expression of (A) OLR1 and (B) IL17A mRNA in peripheral blood mononuclear cells of patients with FP artery disease compared with healthy controls. OLR1, oxidized low-density lipoprotein receptor 1; IL17A, interleukin 17A; FP, femoropopliteal.

Correlation of OLR1 and IL17A gene expression with blood lipid parameters. Potential associations between the expression of OLR1 and IL17A and blood lipid parameters were investigated using Pearson's correlation analysis. LDL and total cholesterol levels were significantly correlated with OLR1 expression $(r=0.507, \mathrm{P}<0.001 ; \mathrm{r}=0.357, \mathrm{P}=0.006$, respectively; Fig. 3A and B) in patients with FP artery disease. Furthermore, OLR1 expression was positively correlated with the levels of triglyceride $(\mathrm{r}=0.463, \mathrm{P}<0.001)$ and fasting glucose $(\mathrm{r}=0.263$, $\mathrm{P}=0.03$; Fig. $3 \mathrm{C}$ and D). However, no significant association was observed between IL17A expression and blood lipid parameters in patients with FP artery disease $(\mathrm{P}>0.05$; results not shown).

Comparison between genotype frequencies of OLRI SNP rs11053646 and IL17A SNPs rs8193037 and rs3819025. The genotype distribution for the SNPs OLR1 rs11053646 as well as IL17A rs8193037 and rs3819025, between patients with FP artery disease and healthy controls is presented in Table II. The distribution of OLR1 rs11053646 as well as IL17A rs8193037 and rs3819025 genotypes was consistent with HWE expectations for patients and controls $(\mathrm{P}>0.05)$.

There was no significant difference in the genotype frequencies of the OLR1 rs11053646 polymorphism between the individuals with FP artery disease and controls $(\mathrm{P}=0.87)$. In addition, no significant difference was observed between the genotype frequencies of the IL17A rs8193037 and rs3819025 polymorphisms between the patients with FP artery disease and healthy controls ( $\mathrm{P}=0.80$ and 0.92 , respectively; Table II).

Multivariate logistic regression analysis of OLRI and ILI7A genes with regard to factors associated with FP artery disease. The potential roles of OLR1 and IL17A mRNA levels in FP artery disease and the possible risk regarding its development associated with OLR1 SNP rs11053646 and IL17A SNPs rs8193037 and rs3819025 were assessed using multiple 

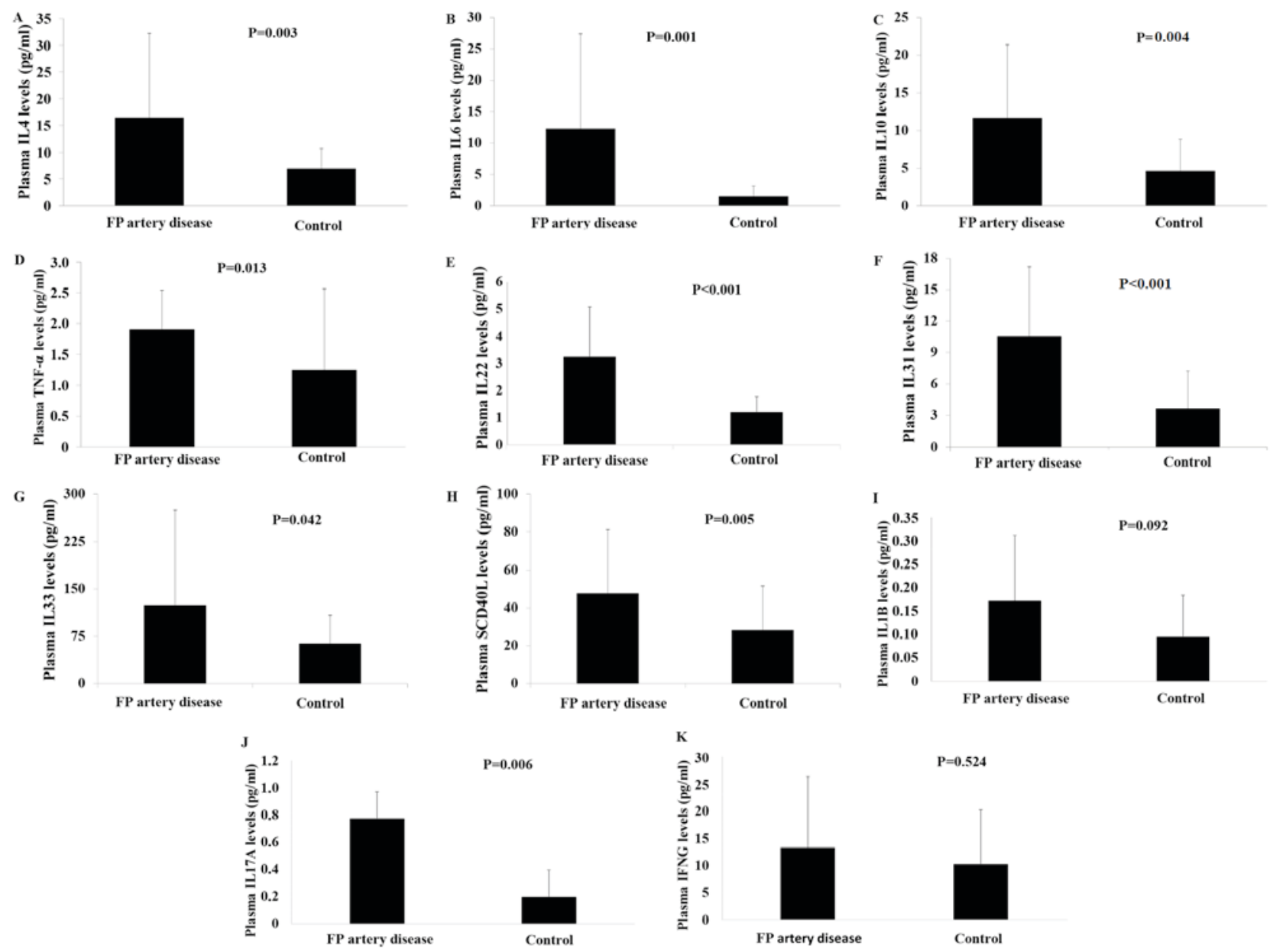

$\mathrm{P}=\mathbf{0 . 5 2 4}$

Figure 2. Levels of plasma cytokines in patients with FP artery disease and healthy controls. (A) Plasma IL4, (B) plasma IL6, (C) plasma IL10, (D) plasma TNF- $\alpha$ levels, (E) plasma IL22, (F) plasma IL31, (G) plasma IL33, (H) plasma SCD40 L, (I) plasma IL1B, (J) plasma IL17A and (K) plasma IFNG. FP, femoropopliteal; IL, interleukin; TNF, tumor necrosis factor; SCD40 L, soluble cluster of differentiation 40 ligand; IFNG, interferon- $\gamma$.

logistic regression analysis with adjustments for a number of factors associated with FP artery disease. These factors, including serum CRP levels $(\mathrm{P}=0.038, \mathrm{OR}=1.587 ; 95 \% \mathrm{CI}$ : 1.025-2.456) together with OLR1 mRNA levels $(\mathrm{P}=0.006)$ were significantly associated with FP artery disease. Furthermore, hypertension $(\mathrm{P}=0.034)$ was significantly associated with FP artery disease. However, neither the OLR1 rs11053646 nor the IL17A rs8193037 and rs3819025 genotypes and gender were not associated with a risk of FP artery disease $(\mathrm{P}>0.05$; Table III).

\section{Discussion}

Atherosclerosis is a multifactorial and multistep disease involving inflammation at all stages from initiation to progression, as well as plaque rupture (19). OLR1 (also known as LOX1) is a membrane protein previously identified in endothelial cells as an oxLDL receptor (20). OLR1 may serve various roles in endothelial dysfunction and proinflammatory signaling (20). In addition, IL17 secreted from Th17 cells, may have diverse roles in various inflammatory diseases (11). IL17 has proatherogenic effects, inducing chemokine, cytokine and matrix metalloproteinase production (21). A number of peripheral arterial diseases, including FP artery disease, are primarily caused by atherosclerosis and it has been determined that OLR1 and IL17 are associated with plaque formation and atherosclerosis $(3,20,21)$. Lim et al (22) demonstrated that atherogenic mice presented with increased serum levels of IL17, which was in turn associated with an increased level of Th17 cells in the secondary lymphoid organs. Furthermore, it was determined that dendritic cell-mediated Th17 polarization by triggering IL6 production was induced by oxLDL uptake (22).

LDL is passed into the subendothelial layer of the artery and oxidized by various biochemical mediators and enzymes, resulting in the production of oxLDL. LDL is recognized by the specific LDL receptor (LDLr); however, oxLDL is recognized by various receptors, including oxLDL receptor-1 (OLR1), cluster of differentiation (CD) 36, Toll-like receptors, scavenger receptors and CD205 (23). OxLDL induces inflammatory mediators and cell adhesion molecules that recruit inflammatory cells and macrophages into the subendothelial layer (24). Arjuman and Chandra (6) evaluated the modulation of OLR1 in the presence of IL10 and determined that oxLDL and IL10 stimulated cell surface expression of OLR1 in the THP-1 macrophage cell line. Ox-LDL-induced OLR1 subsequently promoted intracellular nitric oxide, which acts as a pro-inflammatory substance (6). In the present study, 
Table II. Distribution of OLR1 SNP rs11053646 and IL17A SNPs rs8193037 and rs3819025 genotype and allele frequencies among patients with FP artery disease and healthy controls.

\begin{tabular}{|c|c|c|c|c|c|}
\hline Genotype/allele & FP artery disease $(n=70)$ & Control $(n=80)$ & P-value & OR & $95 \% \mathrm{CI}$ \\
\hline \multicolumn{6}{|l|}{ OLR1 rs11053646 } \\
\hline $\mathrm{CC} ; \mathrm{n}(\%)$ & $61(87.1)$ & $69(86.3)$ & & 0.99 & $0.873-1.122$ \\
\hline $\mathrm{GC}+\mathrm{GG} ; \mathrm{n}(\%)$ & $9(12.9)$ & $11(13.8)$ & 0.87 & 1.069 & $0.471-2.429$ \\
\hline $\mathrm{C}$ allele frequency & 0.93 & 0.92 & & & \\
\hline $\mathrm{G}$ allele frequency & 0.07 & 0.08 & 0.79 & & \\
\hline \multicolumn{6}{|l|}{ IL17A rs8193037 } \\
\hline GG; n $(\%)$ & $61(87.1)$ & $71(88.8)$ & & 1.018 & $0.904-1.147$ \\
\hline $\mathrm{GA}+\mathrm{AA} ; \mathrm{n}(\%)$ & $9(12.9)$ & $9(11.3)$ & 0.80 & 0.875 & $0.368-2.081$ \\
\hline $\mathrm{G}$ allele frequency & 0.94 & 0.94 & & & \\
\hline A allele frequency & 0.06 & 0.06 & 0.86 & & \\
\hline \multicolumn{6}{|l|}{ IL17A rs3819025 } \\
\hline GG; n (\%) & $59(84.3)$ & $67(83.7)$ & & 0.994 & $0.864-1.143$ \\
\hline $\mathrm{GA}+\mathrm{AA} ; \mathrm{n}(\%)$ & $11(15.7)$ & $13(16.3)$ & 0.92 & 1.034 & $0.495-2.159$ \\
\hline $\mathrm{G}$ allele frequency & 0.91 & 0.91 & & & \\
\hline A allele frequency & 0.09 & 0.09 & 0.80 & & \\
\hline
\end{tabular}

$\chi^{2}$ test was performed. OLR1, oxidized low-density lipoprotein receptor 1; IL17A, interleukin 17A; SNPs, single nucleotide polymorphisms; FP, femoropopliteal; OR, odds ratio; CI, confidence interval.
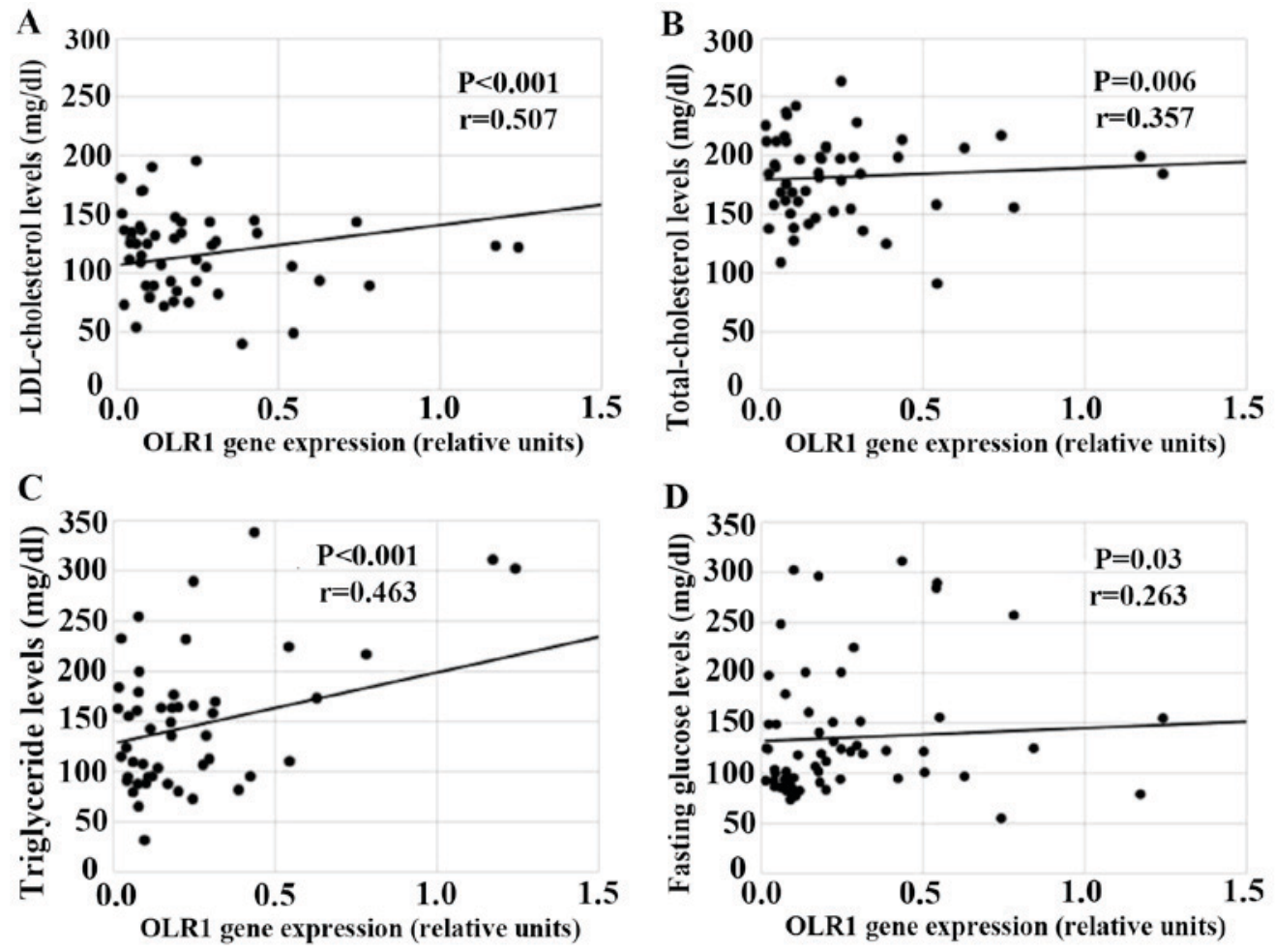

Figure 3. Correlation between expression of OLR1 mRNA and blood lipid parameters in patients with femoropopliteal artery disease. (A) LDL-cholesterol levels, (B) total cholesterol levels, (C) triglyceride levels and (D) fasting glucose levels. OLR1, oxidized low-density lipoprotein receptor 1; LDL, low-density lipoprotein.

a significant increase in hematocrit, fasting glucose, urea, creatinine and serum CRP levels was detected in patients with FP artery disease compared with controls. This suggested the emergence of induced inflammatory pathways in the patient group. However, no significant difference was observed in the total, HDL and LDL cholesterol levels between the control and FP artery groups. This may be attributed to the use of statins by the FP artery disease group of the present study. 
Table III. Logistic regression analysis for FP artery disease risk.

\begin{tabular}{lccc}
\hline Genotype/allele & P-value & $\operatorname{Exp}(\mathrm{B})$ & 95\% CI \\
\hline OLR1 mRNA levels & 0.006 & & \\
Serum CRP levels & 0.038 & 1.587 & $1.025-2.456$ \\
LDL-cholesterol levels & 0.081 & 0.945 & $0.887-1.007$ \\
Hypertension (+) & 0.034 & & \\
Constant & 0.393 & & \\
\hline
\end{tabular}

Adjustments for age, gender, LDL-cholesterol levels, serum CRP levels, OLR1 and IL17A mRNA levels and hypertension status were performed. Exp (B), exponentiation of the B coefficient; CI, confidence interval; OLR1, oxidized low-density lipoprotein receptor 1; CRP, C-reactive protein; LDL, low-density lipoprotein.

OLR1 is a scavenger receptor that mediates the uptake and binding of oxLDL by vascular cells during the progression of atherosclerosis (25). Exposure to oxLDL induces OLR1 expression and further activates the signaling pathways associated with the biological activity of OLR1, such as nuclear factor- $\mathrm{\kappa B}$ (a nuclear factor involved in the signal transduction of inflammation) (25). Previous studies have investigated the changes in soluble OLR1 levels (sOLR1) in atherosclerotic diseases other than CAD. In one previous study, increased serum sOLR1 levels were noted in diabetic patients with PAD compared to those without PAD; these levels were inversely correlated with ABI (26). These findings suggested that the serum OLR1 concentration is associated with ABI and PAD in patients with type 2 diabetes. In addition, the differences in macrophage trafficking among wild-type OLR1 knock-out (KO), LDLr KO and LDLr/OLR1 double KO mice were determined. OLR1 deletion evoked a reduction in macrophage trafficking in the aorta of LDLr KO mice (27). The results of the present study indicated that OLR1 mRNA expression was significantly higher in the group with FP artery disease compared with that in healthy controls. These results are consistent with the findings of Fukui et al (26); however, in the present study, the incidence of type 2 diabetes in the patient group was only $26.5 \%$. It was also observed that factors, including serum CRP levels along with OLR1 mRNA expression and hypertension, were significantly associated with FP artery disease.

To the best of our knowledge, the association between OLR1 SNP rs1 1053646 and peripheral arterial diseases has not yet been studied. In a recent meta-analysis of seven case-control studies, it was observed that OLR1 SNP rs11053646 dominant and co-dominant models were significantly associated with ischemic stroke (28). In the present study, OLR1 SNP rs11053646 recessive and co-dominant models were not significantly associated with the risk of FP artery disease. This result may be due to the limited sample size of the study.

Potekhina et al (29) reported that the anti-atherogenic regulatory $\mathrm{T}$ cell/proatherogenic Th17 cell ratio declined in patients with severe coronary atherosclerosis compared to those with intact coronary artery and coronary artery without atherosclerosis progression (29). It was determined that the imbalance in the pro- and anti-inflammatory/atherogenic lymphocyte subpopulations was associated with the progression of atherosclerosis (29). In addition, it was demonstrated that IL17A deficiency did not affect the aortic plaque burden in mice fed a high-fat diet or subjected to angiotensin II infusion (30).

Mai et al (11) demonstrated that the hyperlipidemia stimulus oxLDL upregulated IL17 receptors in human and mouse primary aortic endothelial cells and that IL17A, in turn, activated human and mouse primary aortic endothelial cells via the upregulation of pro-inflammatory cytokines such as IL6 and granulocyte-macrophage colony-stimulating factor. In another study, atherogenic mice exhibited increased levels of serum IL17 and Th17 cells (22). Pro-atherogenic factors promoted the polarization and inflammatory function of autoimmune $\mathrm{T}$ cells and antibodies directed against oxLDL inhibited cell polarization. OxLDL, but not native LDL, promoted dendritic cell-mediated Th17-cell polarization in atherogenic mice (22). In the present study, IL17A mRNA expression in the PBMCs of patients with FP artery disease was increased compared with that in healthy controls. In addition, OLR1 expression was compared with stratification by gender, which demonstrated that male patients had higher IL17A mRNA levels compared with female patients, however this difference was not statistically significant. Furthermore, plasma cytokine levels in patients with FP artery disease and healthy controls were assessed. It was demonstrated that plasma IL4, $-6,-10,-22,-31$ and -33 as well as SCD40 L and TNF- $\alpha$ levels were significantly higher in patients with FP artery disease compared with controls. IL1B, IL17A and IFNG levels were also increased in the patients with FP artery disease; however, this difference was not significant.

Chronic inflammation in the arterial wall due to the invasion, proliferation and differentiation of leukocytes is an important phenomenon occurring during the development of atherosclerotic lesions. Ge et al (31) reported that impaired renal function increased the atherosclerotic lesion size and aortic leukocyte infiltration. It was also demonstrated that renal impairment and IL17A during myeloid cell differentiation enhanced antigen-presenting cell marker expression and decreased oxLDL uptake. Another study indicated that hypercholesterolemia resulted in increased aortic inflammation and immune response to modified lipids with the increase in splenic Th17-cell population. In addition, the increase in Th17 cells was positively correlated with the progression of atherosclerosis and immunoglobulin $\mathrm{M}$ antibodies specific to oxLDL and Th17 cells were associated with atherosclerosis development (32). In the present study, associations between OLR1 and IL17A expression and blood lipid parameters were investigated and it was demonstrated that LDL and total cholesterol levels were significantly with OLR1 expression in patients with FP artery disease. OLR1 expression was also positively correlated with triglyceride and fasting glucose levels. However, no significant association was observed between IL17A expression and blood lipid parameters in patients with FP artery disease.

Zhang et al (10) reported that the incidence of IL17A rs8193037 GG homozygote and G allele was significantly higher in patients with CAD than in the general Chinese Han population. The $\mathrm{G}$ allele was associated with an increased 
risk of CAD in male patients. In addition, plasma IL17A levels were higher in patients with AMI, and the G allele was associated with increased expression of IL17A in AMI patients and was a predictive factor for CAD. The same study investigated the IL17A rs3819025 polymorphism in patients with CAD and reported no significant difference in the genotype and allele frequencies between patients with CAD and controls (10). In another study, no association was reported between rs8193037 and premature CAD; however, certain haplotypes were involved in determining the risk of developing premature CAD (33). In the present study, no significant difference was observed in the genotype and allele frequencies of IL17A SNP rs8193037 between patients with FP artery disease and controls, consistent with results of a study by Vargas-Alarcón et al (33). However, the results of the present study were not consistent with the findings of Zhang et al (10), which may be attributed to ethnic differences between the populations studied. The role of the IL17A rs3819025 polymorphism in the development of FP artery disease was also investigated and consistent with the finding of Zhang et al (10), as no association between this polymorphism and FP artery disease was observed.

In conclusion, although the sample size of the present study was limited it is, to the best of our knowledge, the first to identify an association between OLR1 and IL17A mRNA expression in PBMCs and FP artery disease. The present study also reported some large standard deviation values, which is another limitation. As FP artery disease has a multifactorial inheritance, it has both genetic and environmental based complex development and many factors may affect its pathogenesis, thus expanding the standard deviation values. The results suggested that OLR1, IL17A and various cytokines may serve a significant role in the inflammatory mechanism involved in the development of FP artery disease and are associated with blood lipid parameters. The susceptibility genes involved in PAD development remain elusive. Future studies should focus on the regulatory non-coding RNAs involved in the formation of oxLDL and its association with IL17.

\section{Acknowledgements}

The present study was supported by the Scientific Research Projects Coordination Unit of Istanbul University (grant nos. 39959 and 46022).

\section{References}

1. Norgren L, Hiatt WR, Dormandy JA, Nehler MR, Harris KA, Fowkes FG; TASC II Working Group, Bell K, Caporusso J, Durand-Zaleski I, et al: Inter-Society consensus for the management of peripheral arterial disease (TASC II). Eur J Vasc Endovasc Surg 33 (Suppl 1): S1-S75, 2007.

2. Serrano Hernando FJ and Martín Conejero A: Peripheral artery disease: Pathophysiology, diagnosis and treatment. Rev Esp Cardiol 60: 969-982, 2007 (In Spanish)

3. Mahameed AA: Peripheral arterial disease. http://www.clevelandclinicmeded.com/medicalpubs/diseasemanagement/cardiology/ peripheral-arterial-disease/. Accessed January 11, 2009.

4. Kullo IJ and Leeper NJ: The genetic basis of peripheral arterial disease: Current knowledge, challenges, and future directions. Circ Res 116: 1551-1560, 2015.

5. Chen XP and DU GH: Lectin-like oxidized low-density lipoprotein receptor-1: Protein, ligands, expression and pathophysiological significance. Chin Med J (Engl) 120: 421-426, 2007.
6. Arjuman A and Chandra NC: Effect of IL-10 on LOX-1 expression, signaling and functional activity: An atheroprotective response. Diab Vasc Dis Res 10: 442-451, 2013.

7. Tesmer LA, Lundy SK, Sarkar S and Fox DA: Th17 cells in human disease. Immunol Rev 223: 87-113, 2008.

8. Taleb S, Tedgui A and Mallat Z: Adaptive T cell immune responses and atherogenesis. Curr Opin Pharmacol 10: 197-202, 2010.

9. Madhur MS, Lob HE, McCann LA, Iwakura Y, Blinder Y, Guzik TJ and Harrison DG: Interleukin 17 promotes angiotensin II-induced hypertension and vascular dysfunction. Hypertension 55: 500-507, 2010.

10. Zhang X, Pei F, Zhang M, Yan C, Huang M, Wang T and Han Y: Interleukin-17A gene variants and risk of coronary artery disease: A large angiography-based study. Clin Chim Acta 412: 327-331, 2011.

11. Mai J, Nanayakkara G, Lopez-Pastrana J, Li X, Li YF, Wang X, Song A, Virtue A, Shao Y, Shan H, et al: Interleukin-17A promotes aortic endothelial cell activation via transcriptionally and post-translationally activating p38 mitogen-activated protein kinase (MAPK) pathway. J Biol Chem 291: 4939-4954, 2016.

12. Biocca S, Falconi M, Filesi I, Baldini F, Vecchione L, Mango R, Romeo F, Federici G, Desideri A and Novelli G: Functional analysis and molecular dynamics simulation of LOX-1 K167N polymorphism reveal alteration of receptor activity. PLoS One 4: e4648, 2009.

13. Tatsuguchi M, Furutani M, Hinagata J, Tanaka T, Furutani Y Imamura S, Kawana M, Masaki T, Kasanuki H, Sawamura T and Matsuoka R: Oxidized LDL receptor gene (OLR1) is associated with the risk of myocardial infarction. Biochem Biophys Res Commun 303: 247-250, 2003.

14. Hou XW, Wang LF, Wang N, Pang D, Hui B, Zhou YL and He X: The G501C polymorphism of oxidized LDL receptor gene [OLR-1] is associated with susceptibility and serum C-reactive protein concentration in Chinese essential hypertensives. Clin Chim Acta 388: 200-203, 2008.

15. Hattori H, Sonoda A, Sato H, Ito D, Tanahashi N, Murata M, Saito I, Watanabe K and Suzuki N: G501C polymorphism of oxidized LDL receptor gene (OLR1) and ischemic stroke. Brain Res 1121: 246-249, 2006.

16. Zhang J, Yin C, Zhang Y, Zhao L, Fu H and Feng J: The role of OLR1 polymorphisms in determining the risk and prognosis of ischemic stroke in a Chinese population. NeuroRehabilitation 32: 391-396, 2013.

17. Liu X, Zhu RX, Li L and He ZY: Association of LOX-1 gene polymorphisms with cerebral infarction in northern Chinese Han population. Lipids Health Dis 13: 55, 2014.

18. Livak KJ and Schmittgen TD: Analysis of relative gene expression data using real-time quantitative PCR and the 2(-Delta Delta C(T)) Method. Methods 25: 402-408, 2001.

19. Libby P, Ridker PM and Maseri A: Inflammation and atherosclerosis. Circulation 105: 1135-1143, 2002.

20. Dunn S, Vohra RS, Murphy JE, Homer-Vanniasinkam S, Walker JH and Ponnambalam S: The lectin-like oxidized low-density-lipoprotein receptor: A pro-inflammatory factor in vascular disease. Biochem J 409: 349-355, 2008.

21. Liuzzo G, Trotta F and Pedicino D: Interleukin-17 in atherosclerosis and cardiovascular disease: The good, the bad, and the unknown. Eur Heart J 34: 556-559, 2013.

22. Lim H, Kim YU, Sun H, Lee JH, Reynolds JM, Hanabuchi S, Wu H, Teng BB and Chung Y: Proatherogenic conditions promote autoimmune $\mathrm{T}$ helper 17 cell responses in vivo. Immunity 40: 153-165, 2014.

23. Goyal T, Mitra S, Khaidakov M, Wang X, Singla S, Ding Z, Liu S and Mehta JL: Current concepts of the role of oxidized LDL receptors in atherosclerosis. Curr Atheroscler Rep 2012 (Epub ahead of print).

24. Hansson GK and Hermansson A: The immune system in atherosclerosis. Nat Immunol 12: 204-212, 2011.

25. Gao S and Geng YJ: LOX-1: A male hormone-regulated scavenger receptor for atherosclerosis. Vascul Pharmacol 59: 138-143, 2013.

26. Fukui M, Tanaka M, Senmaru T, Nakanishi M, Mukai J, Ohki M, Asano M, Yamazaki M, Hasegawa G and Nakamura N: LOX-1 is a novel marker for peripheral artery disease in patients with type 2 diabetes. Metabolism 62: 935-938, 2013.

27. Ding Z, Mizeracki AM, Hu C and Mehta JL: LOX-1 deletion and macrophage trafficking in atherosclerosis. Biochem Biophys Res Commun 440: 210-214, 2013. 
28. Au A, Griffiths LR, Cheng KK, Wee Kooi C, Irene L and Keat Wei L: The influence of OLR1 and PCSK9 gene polymorphisms on ischemic stroke: Evidence from a meta-analysis. Sci Rep 5: 18224, 2015

29. Potekhina AV, Pylaeva E, Provatorov S, Ruleva N, Masenko V, Noeva E, Krasnikova T and Arefieva T: Treg/Th17 balance in stable CAD patients with different stages of coronary atherosclerosis. Atherosclerosis 238: 17-21, 2015.

30. Madhur MS, Funt SA, Li L, Vinh A, Chen W, Lob HE, Iwakura Y, Blinder Y, Rahman A, Quyyumi AA and Harrison DG: Role of interleukin 17 in inflammation, atherosclerosis, and vascular function in apolipoprotein e-deficient mice. Arterioscler Thromb Vasc Biol 31: 1565-1572, 2011

31. Ge S, Hertel B, Koltsova EK, Sörensen-Zender I, Kielstein JT, Ley K, Haller $\mathrm{H}$ and von Vietinghoff S: Increased atherosclerotic lesion formation and vascular leukocyte accumulation in renal impairment are mediated by interleukin-17A. Circ Res 113: 965-974, 2013
32. Rao LN, Ponnusamy T, Philip S, Mukhopadhyay R, Kakkar VV and Mundkur L: Hypercholesterolemia induced immune response and inflammation on progression of atherosclerosis in Apob(tm2Sgy) Ldlr(tm1Her)/J mice. Lipids 50: 785-797, 2015.

33. Vargas-Alarcón G, Angeles-Martínez J, Villarreal-Molina T, Alvarez-León E, Posadas-Sánchez R, Cardoso-Saldaña G, Ramírez-Bello J, Pérez-Hernández N, Juárez-Rojas JG, Rodríguez-Pérez JM, et al: Interleukin-17A gene haplotypes are associated with risk of premature coronary artery disease in Mexican patients from the Genetics of Atherosclerotic Disease (GEA) study. PLoS One 10: e0114943, 2015. 